\title{
The Method of Successive Approximations (Neumann's Series) of Volterra Integral Equation of the Second Kind
}

\author{
Teshome Bayleyegn Matebie \\ College of Natural Science, Department of Mathematics, Arba Minch University, Arba Minch, Ethiopia
}

\section{Email address:}

sbayleyegn130@gmail.com

\section{To cite this article:}

Teshome Bayleyegn Matebie. The Method of Successive Approximations (Neumann's Series) of Volterra Integral Equation of the Second Kind. Pure and Applied Mathematics Journal. Vol. 5, No. 6, 2016, pp. 211-219.doi: 10.11648/j.pamj.20160506.16

Received: October 12, 2016; Accepted: October 28, 2016; Published: December 23, 2016

\begin{abstract}
In this paper, the solving of a class of both linear and nonlinear Volterra integral equations of the first kind is investigated. Here, by converting integral equation of the first kind to a linear equation of the second kind and the ordinary differential equation to integral equation we are going to solve the equation easily. The method of successive approximations (Neumann's series) is applied to solve linear and nonlinear Volterra integral equation of the second kind. Some examples are presented to illustrate methods.
\end{abstract}

Keywords: Volterra Integral Equation, First Kind, Second Kind, Kernel, Method of Successive Approximations

\section{Introduction}

The integral equation originates from the conversion of a boundary-value problem or an initial-value problem associated with a partial or an ordinary differential equation, but many problems lead directly to integral equations and cannot be formulated in terms of differential equations [7], [10] \& [11]. There as on for doing this is that it may make solution of the problem easier, or sometimes enable us, to prove fundamental results on the existence and uniqueness of the solution.

An integral equation is an equation in which the unknown function $f(x)$ to be determined appears under the integral sign [3] \& [7].A typical form of an integral equation in $f(x)$ is of the form.

$$
f(x)=\varphi(x)+\lambda \int_{\alpha(x)}^{\beta(x)} K(x, t) f(t) d t
$$

Where $K(x, t)$ is called the kernel of the integral equation, and $\alpha(x)$ and $\beta(x)$ are the limits of integration. It can be easily observed that the unknown function $\mathrm{f}(\mathrm{x})$ appears under the integral sign. It is to be noted here that both the kernel $K(x, t)$ and the function $\varphi(\mathrm{x})$ in equation are given functions; and $\lambda$ is a constant parameter [1], [3], [5], [7]\&[9].

The prime objective of this paper is to determine the unknown function $f(x)$ that will satisfy equation (1) using a number of Numerical techniques. It needs considerable efforts in exploring these methods to find solutions of the unknown function.

The theory and application of integral equations is an important subject within applied mathematics, physics, and engineering. In particular, they are widely used in mechanics, geophysics, electricity and magnetism, kinetic theory of gases, hereditary phenomena in biology, quantum mechanics, mathematical economics, and queuing theory [2], [7], [8] \& [11].

Integral equations are used as mathematical models for many and varied physical situations, and integral equations also occur as reformulations of other mathematical problems. Now begin with a brief classification of integral equations, and then in later sections, by considering volterra integral equations of the first and the second kind. By doing this, it helps the researchers to prepare themselves for more challenging problems that will be considered in subsequent topics.

\section{Significance of the Study}

Integral equations are often easier to solve, more elegant and compact than a corresponding differential equation. Because it does not require supplementary initial or boundary conditions and the contribution of this study is that:

- Distinguish the importance of integral equation over the differential equation.

- Initiate other researchers for depth study about integral equation using different numerical techniques. 
- It gives clue to extend the concept of integral equation to many interdisciplinary areas instead of using differential equations.

- It invites other researchers interested on the rest part of integral equations.

\section{Research Methods}

In this research, we had used successive approximation method of integral equations in transforming from ordinary differential equation (ODE). Integral equations of the first kind are often extremely ill-conditioned. Applying the kernel to a function is generally a smoothing operation, so the solution, which requires inverting the operator, will be extremely sensitive to small changes or errors in the input.

The treatment of the equation will depend on the smoothness of $\mathrm{K}(\mathrm{x}, \mathrm{t})$ and $\mathrm{f}(\mathrm{x})$. Integral equations of the first kind (linear or nonlinear) are generally suspected of being ill-conditioned or illposed. In such circumstances small changes in $f(x)$ or $K(x, t)$ may have a large effect in the numerical solution of $f(x)$ of the problem. If the original problem had a solution the perturbed equation may have no solution, and vice-versa.

\section{Integral Equations and Their Relationship to Differential Equations}

The theories of ordinary and partial differential equations are a fruitful source of integral equations. The researcher shall sketch here one of the ways in which integral equations can arise from ordinary differential equations. Most ordinary differential equations can be expressed as integral equations, but the reverse is not true [10] \& [11].

To investigate the relationship between integral and deferential equations, The researcher will need the following lemma which will allow us to replace a double integral by a single one.

Lemma 1: (Replacement Lemma) suppose that $f$ : $[a, b] \rightarrow$ IR is continuous. Then

$$
\int_{a}^{x} \int_{a}^{x^{\prime}} f(t) d t d x^{\prime}=\int_{a}^{x}(x-t) f(t) d t, x \in[a, b]
$$

Proof:Define $\mathrm{F}:[\mathrm{a}, \mathrm{b}] \rightarrow \mathrm{IR}$ by

$$
F(x)=\int_{a}^{x}(x-t) f(t) d t, x \in[a, b]
$$

$\operatorname{As}(x-t) f(t)$ and $\frac{\partial}{\partial x}[(x-t) f(t)]$ are continuous for all $x$ and $t$ in $[a, b]$, we can use [Leibniz rule] to differentiate $F$ :

$$
\begin{gathered}
F^{\prime}(x)=(x-t) f(t) l_{t=x} \frac{d}{d x} x+\int_{a}^{x} \frac{\partial}{\partial x}[(x-t) f(t)] d t \\
=\int_{a}^{x} f(t) d t .
\end{gathered}
$$

Since, again by [Leibniz rule], $\int_{\mathbf{a}}^{\mathbf{x}} \mathbf{f}(\mathbf{t}) \mathbf{d t}$ and hence $\frac{\boldsymbol{d} \boldsymbol{F}}{\boldsymbol{d} \boldsymbol{x}}$, are continuous functions of $\boldsymbol{x}$ on $[\mathbf{a}, \mathbf{b}]$, we may now apply the fundamental theorem of calculus I to deduce

$$
F\left(x^{\prime}\right)=F\left(x^{\prime}\right)-F(a)=\int_{a}^{x^{\prime}} F^{\prime}(x) d x=\int_{a}^{x^{\prime}} \int_{a}^{x} f(t) d t d x
$$

Swapping the roles of $x$ and $x^{\prime}$ we have the result as stated. Alternatively, define, for $\left(t, x^{\prime}\right) \in\left[a, x^{\prime}\right]^{2}$

$$
G\left(t, x^{\prime}\right)=\left\{\begin{array}{c}
f(t), \text { whena } \leq t \leq x^{\prime} \leq x \\
0, \text { whena } \leq x^{\prime} \leq t \leq x
\end{array}\right.
$$

The function $G=G\left(t, x^{\prime}\right)$ is continuous, except on the line given by $t=x^{\prime}$, and hence integrable. Using Fubini's Theorem

$$
\begin{gathered}
\int_{a}^{x} \int_{a}^{x^{\prime}} f(t) d t d x^{\prime}=\int_{a}^{x}\left(\int_{a}^{x} G\left(t, x^{\prime}\right) d t\right) d x^{\prime} \\
=\int_{a}^{x}\left(\int_{a}^{x} G\left(t, x^{\prime}\right) d x^{\prime}\right) d t=\int_{a}^{x}\left(\int_{t}^{x} f(t) d x^{\prime}\right) d t= \\
\int_{a}^{x^{\prime}}(x-t) f(t) d t
\end{gathered}
$$

Hence proved.

Now considering the first-order differential equation.

$$
\frac{d y}{d x}=y^{\prime}=f(x, y)
$$

With the initial condition $y(0)=y_{0}$ if say, $f(x, y)$ is continuous function of $(\mathrm{x}, \mathrm{y})$, integrate (5) from 0 tox, obtaining

$$
\begin{aligned}
\int_{0}^{x}\left(\frac{d y}{d x}\right) d x=\int_{0}^{x} f(x, y) d x \int_{0}^{x} d y \\
=\int_{0}^{x} f(t, y(t)) d t y(x)-y(0) \\
=\int_{0}^{x} f(t, y(t)) d t
\end{aligned}
$$

$$
y(x)=y_{0}-\int_{0}^{x} f(t, y(t)) d t, \text { wherey }(0)=y_{0}
$$

This illustrates the general fact that, by going over to integral equations, it includes both the differential equation and the initial conditions in a single equation. Again consider the second-order differential equation.

$$
\frac{d^{2} y}{d^{2}}=y^{\prime \prime}=f(x, y) \text {, With initial conditions. }
$$




$$
y(0)=y_{0}, y^{\prime}=y_{1}
$$

Then integrate (7) from 0 to $\mathrm{x}$. Then

$$
\begin{gathered}
\int_{0}^{x} \frac{d}{d x}\left(\frac{d y}{d x}\right) d x=\int_{0}^{x}[f(t, y(t))] d t \\
y^{\prime}(x)-y^{\prime}(0)=\int_{0}^{x}[f(t, y(t))] d t \\
y^{\prime}(x)=y^{\prime}(0)+\int_{0}^{x}[f(t, y(t))] d t b u t, y^{\prime}(0)=y_{1} \\
y^{\prime}(x)=y_{1}+\int_{0}^{x}[f(t, y(t))] d t
\end{gathered}
$$

Whence the second integration

$$
\begin{gathered}
\int_{0}^{x}\left(\frac{d y}{d x}\right) d x=\int_{0}^{x} y_{1} d t+\int_{0}^{x} d t\left\{\int_{0}^{t}[f(u, y(u))] d u\right\} \\
y(x)-y(0)=y_{1} x+\int_{0}^{x} d t \int_{0}^{t} f(u, y(u)) d u, \operatorname{buty}(0)=y_{0} \\
y(x)=y_{0}+y_{1} x+\int_{0}^{x} d t \int_{0}^{t} f(u, y(u)) d u \\
y(x)=y_{0}+y_{1} x+\int_{0}^{t}[f(t, y(t))] d t \int_{t}^{x} d u \\
y(x)=y_{0}+y_{1} x+\int_{0}^{t}(x-t)[f(t, y(t))] d t
\end{gathered}
$$

The argument is reversible, so that here again the differential equation (7), together with the initial conditions, is equivalent to the single integral equation (9). We see also that any solution of (7) satisfies an integral equation of the form.

$$
y(x)=A+B x+\int_{0}^{t}(x-t)[f(t, y(t))] d t
$$

The constant A and B being determined by the initial conditions. They may also be determined in other ways suppose, for instance, that $\mathrm{y}(\mathrm{x})$ is required to satisfy a two point boundary condition, $\operatorname{say} y(0)=\alpha, y(l)=\beta($ if $x=l$ ) substituting in (10), we obtain.

$$
\begin{aligned}
\alpha=y(0)=A \beta & =y(l) \\
& =A+B l+\int_{0}^{l}(l-t) f[(t, y(t))] d t \\
A=\alpha, B & =\frac{\beta-\alpha}{l}-\frac{1}{l} \int_{0}^{l}(l-t) f[t, y(t)] d t
\end{aligned}
$$

Hence, the function $\mathrm{y}(\mathrm{x})$ must therefore satisfy the integral equation

$$
\begin{aligned}
y(x)=\alpha+ & \frac{\beta-\alpha}{l} x+\int_{0}^{x}(x-t) f[t, y(t)] d t \\
& -\frac{x}{l} \int_{0}^{l}(l-t) f[t, y(t)] d t
\end{aligned}
$$

This can be written in the form

$$
y(x)=z(x)-\int_{0}^{l} K(x, t) f[t, y(t)] d t
$$

Where $z(x)=\alpha+\frac{\beta-\alpha}{l} x$

$$
k(x, t)=\left\{\begin{array}{l}
\frac{t(l-x)}{l}, \text { for } 0 \leq t \leq x \\
\frac{x(l-t)}{l}, \text { for } x \leq t \leq l
\end{array}\right.
$$

$\mathrm{K}(\mathrm{x}, \mathrm{t})$ is the kernel of the equation the argument is again reversible, so that (12) is equivalent to (7) together with the boundary conditions. If the differential equation is linear, we are led in this way to a linear integral equation of the second kind.

\section{Example: 1}

Reduce the initial value problem

$y^{\prime \prime}(x)+4 y(x)=\sin x$, with initial conditions $y(0)=$ $0, y^{\prime}(0)=0$ atx $=0$ to volterra integral equation of the second kind.

\section{Solution:}

Volterra equation can be obtained in the following manner: Let

$$
y^{\prime \prime}(x)=f(x)
$$

Integrate (13) from 0 to $x$. We then have

$$
\begin{gathered}
\int_{0}^{x} \frac{d}{d x}\left(\frac{d y}{d x}\right) d x=\int_{0}^{x} f(t) d t \\
y^{\prime}(x)-y^{\prime}(0)=\int_{0}^{x} f(t) d t \\
y^{\prime}(x)=y^{\prime}(0)+\int_{0}^{x} f(t) d t \\
y^{\prime}(x)=0+\int_{0}^{x} f(t) d t=\int_{0}^{x} f(t) d t
\end{gathered}
$$

Whence the second integration

$$
\begin{gathered}
\int_{0}^{x} \frac{d y}{d x} d x=\int_{0}^{x} \int_{0}^{t} f(u) d u d x \\
=\int_{0}^{x} f(t) d t \int_{t}^{x} d u=\int_{0}^{x}(x-t) f(t) d t
\end{gathered}
$$


Then the given ODE becomes

$$
f(x)+4 \int_{0}^{x}(x-t) f(t) d t=\sin x
$$

This is the Volterra integral equation.

\section{Integral Equation}

Definition: Any equation in which the unknown function $f(x)$ appears under the integral sign and integrals of that function to be solved for $f(x)$ is known as integral equations. The general linear integral equation for the unknown function $f(x)$ is

$$
g(x) f(x)=F(x)+\lambda \int_{\alpha(x)}^{\beta(x)} K(x, t) f(t) d t
$$

$\mathrm{K}(\mathrm{x}, \mathrm{t})$ is called the kernel, $\lambda$ the parameter of the integral equation and $\alpha(\mathrm{x})$ and $\beta(\mathrm{x})$ are constants or $\alpha(\mathrm{x})$ is a constant and $\beta(x)=x$. If $F(x)=0$, then the equation is referred to as homogeneous. Wheng $(x)=0$, the equation is of the first kind; otherwise, it is of the second kind. The kernel is always defined and continuous on

$$
D=\{(x, t): \alpha(x) \leq x \leq \beta(x), \alpha(x) \leq t \leq \beta(x)\}
$$

classification of integral equations.

An integral equation can be classified as a linear or nonlinear integral equation as we know in the ordinary and partial differential equations [4], [6] \& [7]. In the previous section, These have noticed that the differential equation can be equivalently represented by the integral equation. Therefore, there is a good relationship between these two equations. The most frequently used integral equations fall under two major classes, namely Volterra and Fredholm integral equations. Of course, These have to classify them as homogeneous or non homogeneous; and also linear or nonlinear. In some practical problems.

This research is focusing on the Volterra integral equations and its solution by the method of successive approximations (Neumann's series) The classification of integral equations centers on three basic characteristics which together describe their overall structure and it is useful to set these down briefly before entering into greater detail.

I The kind of an equation refers to the location of the unknown function.

- First kindequations have the unknown function present under the integral sign only.

- Secondkind equations also have the unknown function outside the integral.

II The historical descriptions Fredholm and Volterra are concerned with the integration.interval. In a Fredholm equation the integral is over a finite interval with fixed endpoints. In a Volterra equation the integral is indefinite.

IIIThe adjective singular is sometimes used when the integration is improper, either. because the interval is infinite, or because the integrand is unbounded within the. given interval. Obviously an integral equation can be singular on both counts.

\section{Volterra Integral Equation}

Definition: Volterra equations are written in a form where the upper limit of integration $\beta(x)=x$ (independent variable). The most standard form of Volterra linear integralequations is given by the form.

$$
g(x) f(x)=F(x)+\lambda \int_{\alpha(x)}^{x} K(x, t) f(t) d t
$$

- Volterra integral equation of the first kind. $\operatorname{Ifg}(\mathrm{x})=0$, then (15) yields

$$
0=F(x)+\lambda \int_{\alpha(x)}^{x} K(x, t) f(t) d t
$$

This equation is called Volterra first kind integral equations.

- Homogeneous volterra integral equations. If $\mathrm{g}(\mathrm{x})=$ 1 and $\mathrm{F}(\mathrm{x})=0$, then eqn. (15) gives.

$$
f(x)=\lambda \int_{\alpha(x)}^{x} K(x, t) f(t) d t
$$

- Volterra integral equation of the second kind, If the function $\mathrm{g}(\mathrm{x})=1$, then (15)yields.

$$
f(x)=F(x)+\lambda \int_{\alpha(x)}^{x} K(x, t) f(t) d t
$$

This equation is called Volterra integral equations of second kind

- Non homogeneous volterra integral equations. If $\mathrm{g}(\mathrm{x})=1$ and $\mathrm{F}(\mathrm{x}) \neq 0$, hen eqn.(8) gives

$$
f(x)=F(x)+\lambda \int_{\alpha(x)}^{x} K(x, t) f(t) d t
$$

This equation is called non homogeneous Volterra integral equations of second kind For non homogeneous Volterra integral equations $\lambda$ is numerical parameter, whereas for homogeneous Volterra integral equations $\lambda$ is an eigen value parameter because in such a case the integral equation presents an eigen value problem in which the objective is to determine those values of $\lambda$, called the eigenvalues for which the integral equation possesses nontrivial solutions called eigen functions.

\subsection{Kernel of an Integral Equation}

When considering numerical methods for integral equations, particular attention should be paid to the character of the kernel, which is usually the main factor governing the choice of an appropriate quadrature formula or system of approximating functions. Various commonly occurring types of singularity call for individual treatment.

Likewise provision can be made for cases of symmetry, periodicity or other special structure, where the solution may have special properties and/or economies may be affected in the solution process. We note in particular the following cases to which we shall often have occasion to refer in the description of individual algorithms. The presence of the kernel under the operator makes the 
behavior of these equations less transparent than differential equations. Consider the apparently benign kernel clearly the form of the kernel is crucial to nature of the solution, indeed, to its very existence.

- A linear integral equation with a kernel $\mathrm{k}(\mathrm{x}, \mathrm{t})=$ $\mathrm{k}(\mathrm{t}, \mathrm{x})$ is said to be symmetric. This property plays a key role in the theory of fredholm integral equations.

- If $K(x, t)=K(a+b-x, a+b-t)$ in a linear integral equation, the kernel is called centro-symmetric

- If the equations of the kernel has the form $K(x, t)=$ $\mathrm{K}(\mathrm{x}, \mathrm{t}, \mathrm{y}(\mathrm{t}))=\mathrm{K}(\mathrm{x}-\mathrm{t}) \mathrm{g}(\mathrm{t}, \mathrm{y}(\mathrm{t}))$, the equation is called a convolution integral equation; in the linear case $\mathrm{K}(\mathrm{x}-\mathrm{t}) \mathrm{g}(\mathrm{t}, \mathrm{y}(\mathrm{t}))=\mathrm{y}(\mathrm{t})$.

- If the kernel has the form $\mathrm{K}(\mathrm{x}, \mathrm{t}, \mathrm{y}(\mathrm{t}))=\mathrm{K}_{1}(\mathrm{x}, \mathrm{t}, \mathrm{y}(\mathrm{t})), \mathrm{a} \leq \mathrm{t} \leq \mathrm{x}$.

$$
\mathrm{K}(\mathrm{x}, \mathrm{t}, \mathrm{y}(\mathrm{t}))=\mathrm{K}_{2}(\mathrm{x}, \mathrm{t}, \mathrm{y}(\mathrm{t})), \mathrm{x} \leq \mathrm{t} \leq \mathrm{b}
$$

Where the functions $K_{1}$ and $K_{2}$ are well behaved.

\subsection{The Conversion First Kind Integral Equations to the Second Kind Integral Equations}

Thus it would appear that Volterra integral equations of the second kind are more well behaved that Volterra integral equation of the first kind [7]. To the extent that this is true, we may replace any Volterra integral equation of the first kind with Volterra integral equations of the second kind. The relation between Volterra integral equations of the first and the second kind can be established in the following manner. The first kind Volterra equation is usually.

$$
f(x)=\int_{0}^{x} k(x, t) u(t) d t
$$

if the derivatives $\frac{d f}{d x}=f^{\prime}(x), \frac{d K}{d x}=K_{x}(x, t)$ and $\frac{d K}{d t}=$ $K_{t}(x, t)$ exists and are continuous, then the equation can be reduced to one of the second kind into ways.

- The first and the simplest way are to differentiate both sides of equation (11) with respect to $\mathrm{x}$ and we obtain by using the Leibnitz rule.

Leibniz General Rule

If

$$
\begin{gathered}
\mathrm{I}(\mathrm{x})=\int_{\mathrm{v}(\mathrm{x})}^{\mathrm{u}(\mathrm{x})} \mathrm{f}(\mathrm{x}, \mathrm{t}) \mathrm{dt} \text {, then } \mathrm{I}^{\prime}(\mathrm{x})=\frac{\mathrm{d}}{\mathrm{dx}}\left[\int_{\mathrm{v}(\mathrm{x})}^{\mathrm{u}(\mathrm{x})} \mathrm{f}(\mathrm{x}, \mathrm{t}) \mathrm{dt}\right] \\
=\int_{\mathrm{v}(\mathrm{x})}^{\mathrm{u}(\mathrm{x})} \frac{\partial}{\partial \mathrm{x}}(\mathrm{f}(\mathrm{x}, \mathrm{t})) \mathrm{dt} \\
=\int_{v(x)}^{u(x)} \frac{\partial f(x, t)}{\partial x} d t+f(u(x), x) u^{\prime}(x)-f(v(x), x) v^{\prime}(x), \\
\frac{d(f(x)}{d x}=\int_{0}^{x} \frac{\partial}{\partial x}(k(x, t) u(t))
\end{gathered}
$$

$$
\begin{aligned}
& f^{\prime}(x)=\int_{0}^{x} \frac{\partial}{\partial x}(k(x, t) u(t))+K(x, x) u(x) x^{\prime} \\
& \quad-K(0, x) u(x)(0)^{\prime} \\
& K(x, x) u(x)+\int_{0}^{x} K_{x}(x, t) u(t) d t=f^{\prime}(x)
\end{aligned}
$$

If $K(x, x) \neq 0$, then dividing throughout by this we obtain.

$$
\begin{gathered}
K(x, x) u(x)+\int_{0}^{x} K_{x}(x, t) u(t) d t=f^{\prime}(x) \\
u(x)+\int_{0}^{x} \frac{K_{x}(x, t)}{K(x, x)} u(t) d t=\frac{f^{\prime}(x)}{K(x, x)}
\end{gathered}
$$

And the reduction is accomplished. Thus, we can use the method already given above. If the kernel in (21) is squareintegrable, and $\left[\frac{f^{\prime}(x)}{K(x, x)}\right] \in L_{2}[0,1]$ will have a unique solution in $\mathrm{L}_{2}[0,1]$.

The second way to obtain the second kind Volterra integral equation from the first kind is by using integration by parts, if we set.

$$
\int_{0}^{x} u(t) d t=\varnothing(x)
$$

Or equivalently

$$
\int_{0}^{t} u(\varepsilon) d \varepsilon=\varnothing(t)
$$

Then by using integration by parts,

$$
\left[K(x, t) \int_{0}^{t} u(\varepsilon) d(\varepsilon)\right]_{t=0}^{x}-\int_{0}^{x} K_{t}(x, t)\left(\int_{0}^{t} u(\varepsilon) d(\varepsilon)\right) d t=f(x)
$$

which reduces to

$$
[K(x, t) \emptyset(t)]_{t=0}^{x}-\int_{0}^{x} K_{t}(x, t) \emptyset(t) d t=f(x)
$$

And finally we get

$$
K(x, x) \emptyset(x)-K(x, 0) \emptyset(0)-\int_{0}^{x} K_{t}(x, t) \emptyset(t) d t=f(x)
$$

It is obvious that $\varphi(0)=0$, and dividing out by $\mathrm{K}(\mathrm{x}, \mathrm{x})$ we have

$$
\varnothing(x)=\left\{\frac{f^{\prime}(x)}{K(x, x)}\right\}+\int_{0}^{x}\left\{\frac{K_{x}(x, t)}{K(x, x)}\right\} \emptyset(t) d t F(x) \int_{a}^{x} G(x, t) \emptyset(t) d t
$$

where

$$
F(x)=\frac{f(x)}{K(x, x)}, G(x, t)=\frac{K_{t}(x, t)}{K(x, x)}
$$

For this second process it is apparently not necessary for $f(x)$ to be differentiable However, the function $u(x)$ must finally be calculated by differentiating the function $\varphi(x)$ given by the formula.

$$
\varnothing(x)=\left\{\frac{f^{\prime}(x)}{K(x, x)}\right\}+\int_{0}^{x} H(x, t ; 1)\left\{\frac{f^{\prime}(t)}{K(t, t)}\right\} d t
$$


Where $H(x, t: 1)$ is the resolvent kernel corresponding to $\frac{K_{t}(x, t)}{K(x, x)}$. To do this $\mathrm{f}(\mathrm{x})$ must be differentiable

\subsection{Solution of Volterra Integral Equation}

In the previous sections, we have clearly defined the integral equations with some useful illustrations. This section deals with the Volterra integral equations and their solution techniques. The approach of Volterra equations in much the same way as it has been done Fredholm equations, but there is the problem that the upper limit of the integral is the independent variable of the equation. For thus choose a quadrature scheme that utilizes the endpoints of the interval; otherwise we will not be able to evaluate the functional equation at the relevant quadrature points. One could adopt the view that Volterra equations are, in general, just special cases of Fredholm integral equation equations.

$$
f(x)=\varphi(x)+\lambda \int_{0}^{x} K(x, t) f(t) d t
$$

Where $K(x, t)$ is the kernel of the integral equation, $\varphi(x)$ a continuous function of $x$, and $\lambda$ a parameter. Here, $\varphi(x)$ and $\mathrm{K}(\mathrm{x}, \mathrm{t})$ are the given functions but $\mathrm{f}(\mathrm{x})$ is an unknown function that needs to be determined. The limits of integral for the Volterra integral equations are functions ofx. The nonhomogeneous Volterra integral equation of the first kind is defined as

$$
\varphi(x)=\int_{0}^{x} K(x, t) f(t) d t
$$

The important class of integral equations in which many features of the general theory already appeared in the introduction. There are a host of solution techniques to deal with the Volterra integral equations.

\subsubsection{The Method of Successive Approximations for Linear Volterra Integral Equations}

In this method, replace the unknown function $\mathrm{f}(\mathrm{x})$ under the integral sign of the Volterra equation by any selective real-valued continuous function $\mathrm{f}_{0}(\mathrm{t})$, called the zeroth approximation [10]. This substitution will give the first approximation $\mathrm{f}_{1}(\mathrm{t})$ by.

$$
f_{1}(x)=\varphi(x)+\lambda \int_{0}^{x} K(x, t) f_{0}(t) d t
$$

It is obvious that $\mathrm{f}_{1}(\mathrm{x})$ is continuous if $\varphi(\mathrm{x}), \mathrm{K}(\mathrm{x}, \mathrm{t})$, and $\mathrm{f}_{0}(\mathrm{x})$ are continuous. The second approximation $\mathrm{f}_{2}(\mathrm{x})$ can be obtained similarly by replacing $\mathrm{f}_{0}(\mathrm{x})$ in equation (31) by $\mathrm{f}_{1}(\mathrm{x})$ obtained above. And we find

$$
f_{2}(x)=\varphi(x)+\lambda \int_{0}^{x} K(x, t) f_{1}(t) d t
$$

Continuing in this manner, we obtain an infinite sequence of function $\mathrm{f}_{0}(\mathrm{x}), \mathrm{f}_{1}(\mathrm{x}), \mathrm{f}_{2}(\mathrm{x}), \mathrm{f}_{3}(\mathrm{x}), \mathrm{f}_{\mathrm{n}}(\mathrm{x})$,

That satisfies the recurrence relation

$$
f_{n}(x)=\varphi(x)+\lambda \int_{0}^{x} K(x, t) f_{n-1}(t) d t
$$

for $\mathrm{n}=1,2,3, \ldots$ and $\mathrm{f}_{0}(\mathrm{x})$ is equivalent to any selected realvalued function. The most commonly selected function forf $_{0}(\mathrm{x})$ are 0,1 , and $\mathrm{x}$. Thus, at the limit, the solution $\mathrm{f}(\mathrm{x})$ of the equation (29) is obtained as

$$
f(x)=\lim _{n \rightarrow \infty} f_{n}(x)
$$

so that the resulting solution $\mathrm{f}(\mathrm{x})$ is independent of the choice of the zeroth approximation $\mathrm{f}_{0}(\mathrm{x})$. This process of approximation is extremely simple. However, if we follow the Picard's successive approximation method, it needs to set $\mathrm{f}_{0}(\mathrm{x})=\varphi(\mathrm{x})$, and determine $\mathrm{f}_{1}(\mathrm{x})$ and other successive approximation as follows:

$$
\begin{array}{r}
f_{1}(x)=\varphi(x)+\lambda \int_{0}^{x} K(x, t) f_{0}(t) d t f_{2}(x)=\varphi(x)+ \\
\lambda \int_{0}^{x} K(x, t) f_{1}(t) d t f_{n-1}(x)= \\
\varphi(x)+\lambda \int_{a}^{x} K(x, t) f_{n-2}(t) d t f_{n}(x)=\varphi(x)+ \\
\lambda \int_{0}^{x} K(x, t) f_{n-1}(t) d t
\end{array}
$$

The last equation is the recurrence relation. Consider

$$
\begin{gathered}
f_{2}(x)-f_{1}(x) \lambda \int_{0}^{x} K(x, t)\left[\varphi(t)+\lambda \int_{0}^{t} K(t, \tau) \varphi(\tau) d \tau\right] d t- \\
\lambda \int_{0}^{x} K(x, t) \varphi(t) d t=\lambda^{2} \int_{0}^{x} K(x, t) \int_{0}^{t} K(t, \tau) \varphi(\tau) d \tau d t= \\
\lambda^{2} g_{2}(x)
\end{gathered}
$$

Where

$$
g_{2}(x)=\int_{0}^{x} K(x, t) \int_{0}^{t} K(t, \tau) \varphi(\tau) d \tau d t
$$

Thus, it can be easily observed from equation (36) that

$$
f_{n}(x)=\sum_{m=0}^{n} \lambda^{m} g_{m}(x)
$$

If $\mathrm{g}_{0}(\mathrm{x})=\varphi(\mathrm{x})$, and further that

$$
g_{m}(x)=\int_{0}^{x} K(x, t) g_{m-1}(t) d t
$$

Where $\mathrm{m}=1,2,3$, and hence $\mathrm{g}_{1}(\mathrm{x})=\int_{0}^{\mathrm{x}} \mathrm{K}(\mathrm{x}, \mathrm{t}) \varphi(\mathrm{t}) \mathrm{dt}$

The repeated integrals in equation (37) may be considered as a double integral over the triangular region indicated in Figure 1; thus interchanging the order of.

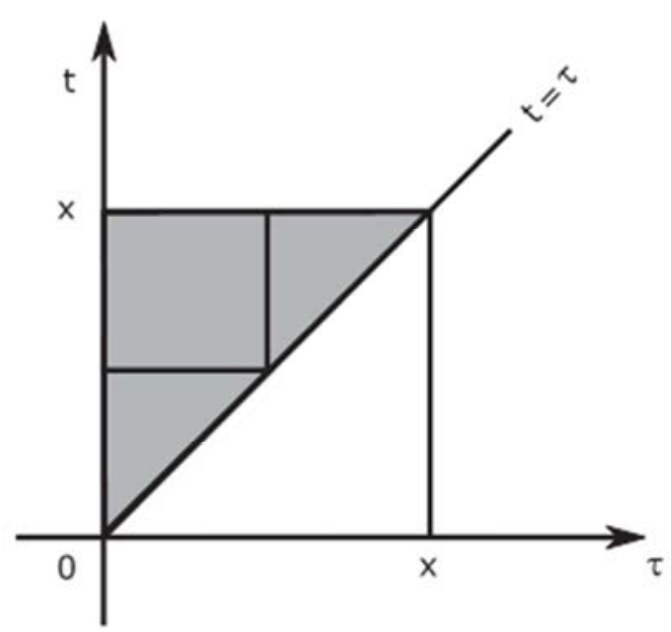

Figure 1. Double integration over the triangular region (shaded area). Integration, we obtain. 
$g_{2}(x)=\int_{0}^{x} \varphi(\tau) d \tau \int_{\tau}^{x} K(x, t) K(t, \tau) d t=\int_{0}^{x} K_{2}(x, \tau) \varphi(\tau) d \tau$

$K_{2}(x, \tau)=\int_{\tau}^{x} K(x, t) K(t, \tau) d t$. Similarly, we find in general.

$$
g_{m}(x)=\int_{0}^{x} K_{m}(x, \tau) \varphi(\tau) d \tau, m=1,2,3,
$$

Where the iterative kernels $K_{1}(x, \tau) \equiv K(x, t), K_{2}(x, t), K_{3}(x, \tau), \ldots$. are defined by the recurrence formula

$$
K_{m+1}(x, t)=\int_{t}^{x} K_{2}(x, \tau) K_{m}(\tau, t) d \tau
$$

Thus, the solution forf $_{\mathrm{n}}(\mathrm{x})$ can be written as

$$
\begin{array}{r}
f_{n}(x)=\varphi(x)+\sum_{m=1}^{n} \lambda^{m} \int_{0}^{x} K_{m}(x, \tau) \varphi(\tau) d \tau \\
\quad=\varphi(x)+\int_{0}^{x}\left\{\sum_{m=1}^{n} \lambda^{m} K_{m}(x, \tau)\right\} \varphi(\tau) d \tau
\end{array}
$$

Hence it is also plausible that the solution of equation (29) will be given be as $n \rightarrow \infty$

$$
\begin{aligned}
& \lim _{\mathrm{n} \rightarrow \infty} \mathrm{f}_{\mathrm{n}}(\mathrm{x})=\mathrm{f}(\mathrm{x})=\varphi(x)+\int_{0}^{x}\left\{\sum_{m=1}^{n} \lambda^{m} K_{m}(x, \tau)\right\} \varphi(\tau) d \tau \\
& =\varphi(x)+\lambda \int_{0}^{x} H(x, \tau ; \lambda) \varphi\left(\varphi(x)+\lambda \int_{0}^{x} e^{x-t} \varphi(t) d t \tau\right) d \tau
\end{aligned}
$$

Where

$$
H(x, \tau ; \lambda)=\sum_{m=1}^{n} \lambda^{m} K_{m}(x, \tau)
$$

is known as the resolvent kernel.

Example 1

Solve the following Volterra integral equation of the second kind of the convolution type using successive approximation method.

$$
f(x)=\varphi(x)+\lambda \int_{0}^{x} e^{x-t} f(t) d t
$$

\section{Solution:}

- using successive approximation method

Let us assume that the zeroth approximation is

$$
f_{0}(x)=0
$$

Then the first approximation can be obtained as

$$
f_{1}(x)=\varphi(x)
$$

Using this information in equation (46), the second approximation is given by.

$$
\begin{aligned}
& f_{2}(x)=\varphi(x)+\lambda \int_{0}^{x} e^{x-t} f_{1}(t) d t \\
& f_{2}(x)=\varphi(x)+\lambda \int_{0}^{x} e^{x-t} \varphi(t) d t
\end{aligned}
$$

Proceeding in this manner, the third approximation can be obtained as

$$
\begin{aligned}
f_{3}(x) & =\varphi(x)+\lambda \int_{0}^{x} e^{x-t} f_{2}(x) d t \\
& =\varphi(x) \\
& +\lambda \int_{0}^{x} e^{x-t}\left\{\varphi(t)+\lambda \int_{0}^{t} e^{t-\tau} \varphi(\tau) d \tau\right\} d t
\end{aligned}
$$

$$
\begin{aligned}
& =\varphi(x)+\lambda \int_{0}^{x} e^{x-t} \varphi(t) d t+\lambda^{2} \int_{0}^{x} \int_{0}^{t} e^{x-\tau} \varphi(\tau) d \tau d t \\
& =\varphi(x)+\lambda \int_{0}^{x} e^{x-t} \varphi(t) d t+\lambda^{2} \int_{0}^{x}(x-t) e^{x-t} \varphi(t) d t
\end{aligned}
$$

In the double integration the order of integration is changed to obtain the final result. In a similar manner, the fourth approximation $\mathrm{f}_{4}(\mathrm{x})$ can be at once written as.

$$
\begin{aligned}
f_{4}(x)=\varphi(x)+ & \lambda \int_{0}^{x} e^{x-t} \varphi(t) d t+\lambda^{2} \int_{0}^{x}(x-t) e^{x-t} \varphi(t) d t \\
& +\lambda^{3} \int_{0}^{x} \frac{(x-t)^{2}}{2 !} e^{x-t} \varphi(t) d t
\end{aligned}
$$

Thus, continuing in this manner, we obtain as $\mathrm{n} \rightarrow \infty$

$$
\begin{aligned}
f(x)=\lim _{n \rightarrow \infty} f_{n}(x) & \\
& =\varphi(x) \\
& +\lambda\left\{\int _ { 0 } ^ { x } e ^ { x - t } \left(1+\lambda(x-t)+\lambda^{2}(x-t)^{2}\right.\right. \\
& +\cdots) \varphi(t) d t\} \\
& =\varphi(x)+\lambda \int_{x}^{x} e^{x-t} e^{\lambda(x-t)} \varphi(t) d t \varphi(x) \\
& +\lambda \int_{0}^{x} e^{(1+\lambda)(x-t)} \varphi(t) d t
\end{aligned}
$$

Here, the resolvent kernel is

$$
H(x, t ; \lambda)=e^{(1+\lambda)(x-t)}
$$

- Another method to determine the solution by the resolvent kernel

The procedure to determine the resolvent kernel is the following: Given that

$$
f(x)=\varphi(x)+\lambda \int_{0}^{x} e^{x-t} f(t) d t
$$


Here, the kernel is $K(x, t)=e^{x-t}$. The solution by the successive approximation is.

$$
f(x)=\varphi(x)+\lambda \int_{0}^{x} H(x, \tau ; \lambda) f(t) d t
$$

where the resolvent kernel is given by

$$
H(x, \tau ; \lambda)=\sum_{n=0} \lambda^{n} K_{n+1}(x, t)
$$

In which

$$
K_{n+1}(x, t)=\varphi(x)+\lambda \int_{t}^{x} K(x, \tau) K_{n}(x, \tau) f(\tau) d \tau, n=1,2,3
$$

It is to be noted that $K_{n}(x, t)=K(x, t)$ Thus, we obtain

$$
K_{2}(x, t)=\int_{t}^{x} e^{x-\tau} e^{(x-\tau)} d \tau=e^{x-t} \int_{t}^{x} d \tau=(x-t) e^{x-t}
$$

Similarly, proceeding in this manner, we obtain

$$
\begin{gathered}
K_{3}(x, t)=\int_{t}^{x} e^{x-\tau}\left(e^{(x-\tau)}(\tau-t)\right) d \tau=e^{x-t} \frac{(x-t)^{2}}{2 !} \\
K_{4}(x, t)=e^{x-t} \frac{(x-t)^{3}}{3 !}= \\
K_{n+1}(x, t)=e^{x-t} \frac{(x-t)^{n}}{n !}
\end{gathered}
$$

Hence the resolvent kernel is

$$
\begin{gathered}
H(x, \tau ; \lambda)=\sum_{n=0} \lambda^{n} K_{n+1}(x, t)=e^{x-t} \sum_{n=0}^{\infty} \frac{(x-t)^{n}}{n !} H(x, t ; \lambda) \\
=e^{(1+\lambda)(x-t)}
\end{gathered}
$$

Once the resolvent kernel is known therefore succeeded in inverting the integral equation because the right- hand side of the above formula is a known quantity.

\subsubsection{The Method of Successive Approximations for Non-Linear Integral Equations}

Nonlinear integral equations yield a considerable amount of difficulties. However, due to recent development of novel techniques it is now possible to find solutions of some types of nonlinear integral equations if not all. In general, the solution of the nonlinear integral equation is not unique. However, the existence of a unique solution of nonlinear integral equations with specific conditions is possible.

We first define a nonlinear integral equation in general, and then cite some particular types of nonlinear integral equations. In general, a nonlinear integral equation is defined as given in the following equation:

$$
\begin{gathered}
f(x)=F(x)+\lambda \int_{\alpha(x)}^{x} K(x, t) G(f(t)) d t \operatorname{orf}(x) \\
=F(x)+\lambda \int_{\alpha(x)}^{x} G(x, t, f(t)) d t
\end{gathered}
$$

Where $\alpha(\mathrm{x})$ and $\beta(\mathrm{x})$ are constants and the equations are called non linear Fredholm integral equations.

$$
\begin{aligned}
f(x)=F(x)+\lambda & \int_{\alpha(x)}^{x} K(x, t) G(f(t)) d t \operatorname{orf}(x) \\
& =F(x)+\lambda \int_{\alpha(x)}^{x} G(x, t, f(t)) d t
\end{aligned}
$$

Where $\alpha(\mathrm{x})$ is constant and $\mathrm{x}$ is independent variable the equations are called non linear Volterra integral equations. The function $G(f(t))$ is non-linear except $G=a$ constant or

$\mathrm{G}(\mathrm{f}(\mathrm{x}))=\mathrm{f}(\mathrm{x})$ in which case $\mathrm{G}$ is linear. $\mathrm{G}(\mathrm{f}(\mathrm{x}))=$ $\mathrm{f}^{\mathrm{n}}(\mathrm{x})$, forn $\geq 2$, then function $\mathrm{G}$ is non linear.

Example 1

$$
f(x)=x+\lambda \int_{0}^{x}(x-t) f^{2}(t) d t
$$

and

$$
(\mathrm{x})=1+\lambda \int_{0}^{\mathrm{x}} \mathrm{K}(\mathrm{x}, \mathrm{t}) \ln (\mathrm{f}(\mathrm{t})) \mathrm{dt}
$$

Are non linear volterra integral equations. By contrast, solving nonlinear Volterra equations usually involves only a slight modification of the algorithm for linear equations.

The Picard's method to obtain successive algebraic approximations. By putting numbers in these, we generally get excellent numerical results. Unfortunately, the method can only be applied to a limited class of equations, in which the successive integrations be easily performed. We shall treat everal examples by these methods to enable their merits to be compared.

Consider the initial value problem given by the first-order nonlinear differential equation.

$\mathrm{df} / \mathrm{dx}=\varphi(\mathrm{x}, \mathrm{f}(\mathrm{x}))$ with the initial condition $\mathrm{f}(\mathrm{a})=$ batx $=$ a.This initial value problem can be transformed to the nonlinear integral equation and is written as

$$
f(x)=b+\int_{a}^{x} \varphi(x, f(t)) d t
$$

For a first approximation, we replace the $\mathrm{f}(\mathrm{x})$ in $\varphi(\mathrm{x}, \mathrm{f}(\mathrm{x}))$ byb, for a second approximation, we replace it by the first approximation, for the third by the second, and so on.

$$
f_{0}(x)=b f_{1}(x)=b+\int_{a}^{x} \varphi(x, b) d t
$$




$$
\begin{gathered}
f_{2}(x)=b+\int_{a}^{x}\left[b+\int_{a}^{x} \varphi(t, b) d t\right] d t \\
f_{2}(x)=b+\int_{a}^{x} b d x+\int_{a}^{x} \int_{a}^{x} \varphi(t, b) d t d t
\end{gathered}
$$

To demonstrate this method by examples.

Example 1 Solve the integral equation by Picard's method of successive approximation.

$$
f(x)=\int_{0}^{x}\left(x+f^{2}(t)\right) d t
$$

\section{Solution}

The given differential equation can be written in integral equation form as

$$
f(x)=\int_{0}^{x}\left(x+f^{2}(t)\right) d t
$$

Zeroth approximation is: $\mathrm{f}(\mathrm{x})=0$.

First approximation: $\operatorname{Put} \mathrm{f}(\mathrm{x})=0$ in $\mathrm{x}+\mathrm{f}^{2}(\mathrm{x})$, yielding

$$
f(x)=\int_{0}^{x} t d t=\frac{1}{2} x
$$

Second approximation:put $f(x)=\frac{x^{2}}{2}$ in $x+f^{2}(x)$, yielding

$$
f(x)=\int_{0}^{x}\left(t+\frac{t^{2}}{2}\right) d t=\frac{x^{2}}{2}+\frac{x^{5}}{20}
$$

Third approximation: $\mathrm{f}=\frac{\mathrm{x}^{2}}{2}+\frac{\mathrm{x}^{5}}{20}$ in $\mathrm{x}+\mathrm{f}^{2}$, giving

$$
\begin{gathered}
f(x)=\int_{0}^{x}\left\{t+\left(\frac{t^{2}}{2}+\frac{t^{5}}{20}\right)^{2}\right\} d t=\int_{0}^{x}\left(t+\frac{t^{4}}{4}+\frac{t^{7}}{20}+\frac{t^{10}}{400}\right) d t \\
=\frac{x^{2}}{2}+\frac{x^{5}}{20}+\frac{x^{8}}{10}+\frac{x^{11}}{4400}
\end{gathered}
$$

Proceeding in this manner, Fourth approximation can be written after a rigorous algebraic manipulation as

Fourth approximation

$$
\begin{gathered}
f(x)=\frac{x^{2}}{2}+\frac{x^{5}}{20}+\frac{x^{8}}{160}+\frac{7 x^{11}}{8800}+\frac{3 x^{14}}{49280}+\frac{x^{20}}{7040000} \\
+\frac{x^{23}}{445280000}
\end{gathered}
$$

and so on. This is the solution of the problem in series form, and it seems from its appearance the series is convergent.

\section{Conclusion}

In thispaper we proposed some of the numerical methods to solve volterra (linear and non linear) integral equations.

$$
\mathrm{f}(\mathrm{x})=\varphi(\mathrm{x})+\lambda \int_{\alpha(\mathrm{x})}^{\mathrm{x}} \mathrm{k}(\mathrm{x}, \mathrm{t}) \mathrm{f}(\mathrm{t}) \mathrm{dt}
$$

And

$$
\mathrm{f}(\mathrm{x})=\varphi(\mathrm{x})+\lambda \int_{\alpha(\mathrm{x})}^{\mathrm{x}} \mathrm{K}(\mathrm{x}, \mathrm{t}) \mathrm{G}(\mathrm{f}(\mathrm{t})) \mathrm{dt}
$$

To solve this for $\mathrm{f}(\mathrm{x})$ the choice of the initial data $\mathrm{f}_{0}(\mathrm{x})$, plays an essential role on the speed of the convergence of the numerical methods, successive approximation method. Numerical methods have been selected based on the ability to obtain rapid convergence, based on existence of reliable and cheaply computable error estimates and personal predilection

When the kernel is simple enough to be closely approximated by a degenerate kernel with a few functions, this can be very efficient. How competitive in general such a method is with the more usual approaches is an unresolved question.

A solution of the integral equation gives more accurate results than a solution of the differential equation using the same step size and degree of precision in the integration procedure.

\section{References}

[1] W. V. Lovitt. (1950). Linear Integral Equations, Dover Represented.

[2] S.S.SASTRY. (2003). Introductory Methods of Numerical Analysis, Third Edition.

[3] M. Rahman. (2007). Integral Equations and their Applications, $1^{\text {st }}$ ed; WIT Press.

[4] Peter Linz. (1985). Analytical and Numerical Methods for Volterra Equations.

[5] Porter, David. (1990). Integral equations A practical treatment, from spectral theory to applications, first edition.

[6] F. Smithies (1958). Cambridge Tracts in Mathematics and Mathematical Physics.

[7] Peter J. Collins. (2006). Differential and Integral equations, WIT Press.

[8] William Squire. (1970). Modern Analytic and Computational Methods in Science and Mathematics.

[9] W. Pogorzelski, Integral Equations and their Applications, volume I.

[10] Abdul-Majid Wazwaz. A First Course in Integral Equations Solutions Manual, $2^{\text {nd }}$ ed; World Scientific Publishing Co. Pte. Ltd.

[11] T. A. Burton Eds. (2005). Volterra Integral and Differential Equations, $2^{\text {nd }}$ ed; Academic Press, Elsevier. 